\title{
A Study of Correlation between Severity of Diastolic Dysfunction and Coronary Angiographic Severity in STEMI Patients
}

\author{
Dr. B. Adilakshmi M.D., D.M ${ }^{1}$, Dr. P. Sudarshan M. D., D.M ${ }^{2}$ \\ ${ }^{I}$ (Associate Professor of Cardilogy, Siddhartha Medical College, Vijayawada, Andhra Pradesh, India.) \\ ${ }^{2}$ (Senior Resident, Cardilogy)
}

\section{Introduction}

The interest in diastolic dysfunction, which is present in various heart diseases, has been growing for many years. Over the past 2 decades, the concept of heart failure with preserved ejection fraction has emerged (1).Ischemic heart disease is one of the main causes of diastolic heart failure. The presence of diastolic dysfunction predicts a poor prognosis in patients with coronary artery disease (2). Heart failure with preserved ejection fraction has become a diagnostic and therapeutic challenge, since its morbidity and mortality are similar to those of heart failure with deteriorated left ventricular ejection fraction (3). Echocardiography is the method of choice for diastolic function testing in clinical practice.

It would be helpful if the severity of the CAD correlated with the degree of abnormal left diastolic function. One could also ascertain the prognosis of the disease, as it has been adequately shown that prognosis is in part dependent on the severity of coronary artery disease (CAD). To evaluate these possibilities, a coronary score was used to assess the degree of CAD. The Aim of this study is to correlate the severity of diastolic dysfunction with the coronary angiographic severity with Leaman's score (4) in those presenting with first STEMI.

\section{Study population:}

\section{Patients And Methods}

The study included 34 patients who are presenting with first STEMI to the ICU cardilogy department, King George Hospital, Andhra medical college, Visakhapatnam. And those underwent coronary angiogram along with a detailed echocardiographic evaluation during the period of 2013-15

\section{Echocardio graphic acquisition and analysis:}

All the participants underwent a detailed echocardiographic evaluation with standard echocardiographic and Doppler recordings, they were performed by an experienced sonographer.

Echocardiography was done using a Philips IE-33 machine equipped with $2.5 \mathrm{MHz}$ transducer. Mitral diastolic inflow was interrogated using pulsed-wave Doppler from the apical 4-chamber view with the sample volume placed at the level of the mitral leaflet tips. Mitral early diastolic peak (E wave), late peak (A wave) velocities, E/A ratio, with tissue doppler imaging septal e1 velocities were also calculated and patients were segregated into four groups according to their diastolic dysfunction severity as grading was done according to American society of echocardiography criteria(5).

\begin{tabular}{|l|l|l|l|l|}
\hline & $\begin{array}{l}\text { Normal diastolic } \\
\text { function }\end{array}$ & $\begin{array}{l}\text { Impaired } \\
\text { relaxation group }\end{array}$ & $\begin{array}{l}\text { Pseudonormalisation } \\
\text { group }\end{array}$ & $\begin{array}{l}\text { Restrictive filling } \\
\text { group }\end{array}$ \\
\hline E/A ratio & $>1$ & $<0.8$ & $0.8-1.6$ & $>2$ \\
\hline E/e1 ratio & $5-10$ & $\leq 8$ & $9-13$ & $>13$ \\
\hline
\end{tabular}

Table 1. Showing the diastolic dysfunction grading according to the American society of echocardiography criteria

All those who are having LBBB in their baseline ECG, a history of cardiomyopathy, severe MR, arrhythmias, EF $<45 \%$, high LV mass during the study were excluded from the study.

\section{Coronary angiographic score (Leaman's score):}

The purpose of the coronary score was to quantitate the severity of the underlying CAD. The different coronary vessels carry different volumes of blood to the left ventricle, and the coronary score was derived to take this into account. A review of the literature revealed the average left ventricular mass in the average male (free wall plus septum) is approximately 155 g.(6), the score was calculated with Leaman's score

The LAD carries approximately 3.5 times and the circumflex 1.5 times as much blood as the right coronary artery. This then was the basis for assigning different weighting values to the different coronary arteries. The coronary arteries were subdivided into segments according to the scheme devised by the American 
A Study Of Correlation Between Severity Of Diastolic Dysfunction And Coronary Angiographic...

Heart Association.(7) The right coronary artery does not supply blood to the left ventricle until the posterior descending branch is given off, so only the segment with the most severe disease was analyzed. However, the LAD and circumflex coronary arteries were further subdivided and weighting factors were assigned to each segment (fig. 1).

Subjects who had a left-dominant coronary artery system were graded differently. In this situation, the left ventricle receives its blood supply from the left coronary artery, so the right coronary artery was not weighted and its value was assigned to the main left coronary artery. Subsequently the circumflex coronary artery was also weighted heavier than in the right-dominant system (fig. 1). The degree of stenosis was also considered in deriving the coronary score. If the vessel was totally occluded, the coronary artery segment value was multiplied by 5.0. If there was a $90-99 \%$ occlusion, it was multiplied by 3.0 . For a $70-89 \%$ obstruction, it was multiplied by 1.0. The segmental scores were then added to derive the total coronary score. A score of zero would indicate no obstructive $\mathrm{CAD}$, and the higher the score, the greater the obstruction (fig. 1)

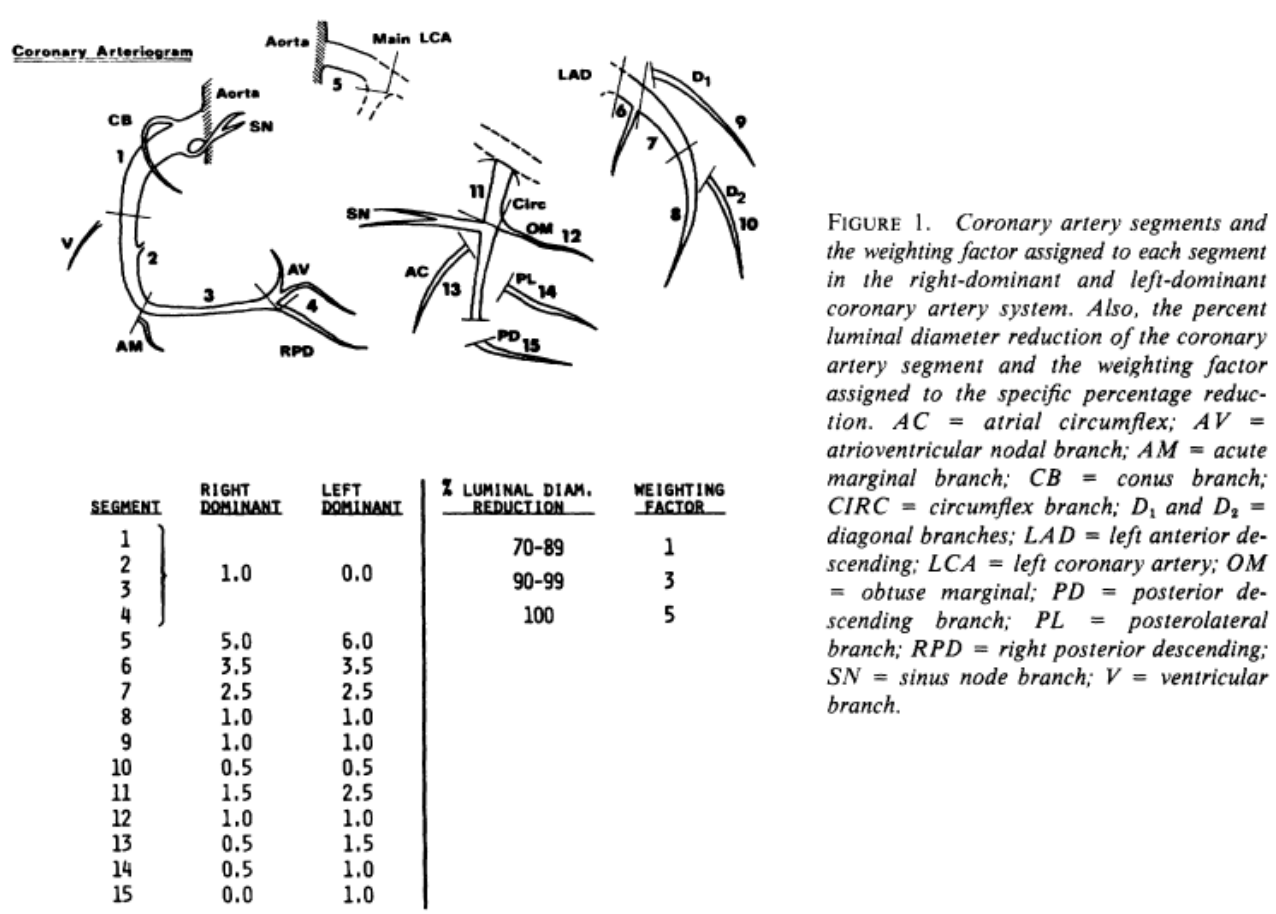

III. Results

The total number of the patients included in the study are 70 patients of whom 34 underwent CAG, the remaining were excluded as they are having severe MR, left ventricular hypertrophy, arrhythmias during the study.

Their baseline characters were shown in the table below Baseline characters table.

\begin{tabular}{|c|c|c|c|c|c|}
\hline & Normal & Impaired relaxation & Pseudo normalisation & Restrictive filling & P value \\
\hline Total & 8 & 6 & 16 & 4 & \\
\hline Male & 6 & 5 & 11 & 3 & 0.07 \\
\hline Female & 2 & 1 & 5 & 1 & 0.09 \\
\hline Mean age & $47.5 \pm 8.34$ & $62.5 \pm 10.25$ & $56.18 \pm 10.17$ & $54.75 \pm 13.96$ & 0.4 \\
\hline HTN & 4 & 3 & 4 & 2 & 0.1 \\
\hline DM & 2 & 1 & 1 & 2 & 0.7 \\
\hline Smoking & 4 & 4 & 10 & 2 & 0.85 \\
\hline Alcoholism & 4 & 3 & 5 & 2 & 0.45 \\
\hline Ant.MI & 4 & 4 & 12 & 2 & 0.65 \\
\hline Inf.MI & 4 & 2 & 4 & 2 & 0.43 \\
\hline $\begin{array}{l}\text { Use of } \\
\text { thrombolytics }\end{array}$ & 5 & 4 & 7 & 3 & $\mathbf{0 . 5 3}$ \\
\hline
\end{tabular}

Table: It is showing the baseline characters of the patients age, gender, Hypertension, diabetes, other risk factors, are almost similar in between the groups 


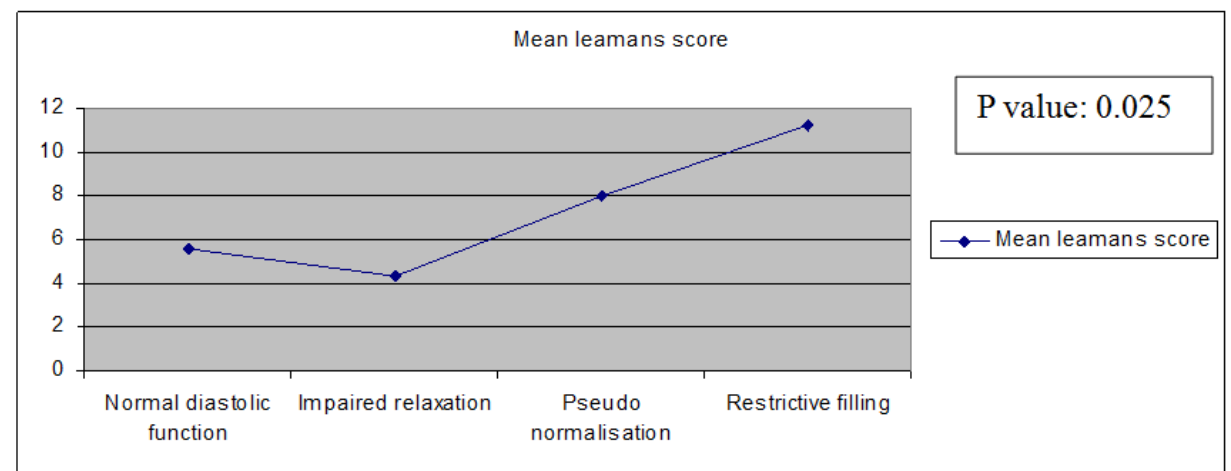

Fig: It showing the mean Leaman's score on the Y axis, severity of diastolic dysfunction on the X axis, as the severity of the diastolic dysfunction increase the Leaman's score also increase.

As the diastolic dysfunction severity is increasing there is a statistically significant increase in the mean leaman's score but for the discrepancy in the first two groups.

\section{Discussion}

The main finding in this study is that as the severity of the diastolic dysfunction increase the angiographic severity also increased in most of the instances except between the normal diastolic function, impaired relaxation group and the differences between groups is also statistically significant.

The reasons for the less severe score in impaired relaxation group when compared to normal group when investigated further was that these patients were found to have either a long LAD ( type IV) which is supplying even the posterior part of the inter ventricular septum but only fixed score is given to lesion in LAD with out taking into consideration the anatomic variability, in a similar manner those who are having lesion in RCA were also found to have large PDA, and also it is giving of many PLV branches but this anatomic variability is not taken into consideration.

\section{Conclusions}

As the severity of the diastolic dysfunction increase the angiographic severity also increase but there are some lacunaes in the leamen's score which does not take into consideration the anatomic variability which is often present in patients inspite of this fact it correlate well with diastolic dysfunction.

\section{References}

[1]. Caudron J, Fares J, Bauer F, Dacher JN. Evaluation of left ventricle diastolic function with cardiac MR imaging. Radio Graph 2011;31:239-61

[2]. Ren X, Na B, Ristow B, et al. Usefulness of diastolic dominant pulmonary vein flow to predict hospitalization for heart failure and mortality in ambulatory patients with coronary heart disease (from the heart and soul study). Am J Cardiol 2009;103(4):482-5.

[3]. Tribouilloy C, Rusinaru D, Mahjoub H, et al. Prognosis of heart failure with preserved ejection fraction: a 5-year prospective population-based study. Eur Heart J 2008;29(3):339-47.

[4]. Coronary Artery Atherosclerosis: Severity of the Disease, Severity of Angina Pectoris and Compromised Left Ventricular Function DAVID M. LEAMAN, M.D., Circulation 63, No. 2, 1981.

[5]. Nagueh SF, Appleton CP, Gillebert TC, Marino PN, Oh JK, Smiseth OAS, et al. Recommendations for the evaluation of left ventricular diastolic function by echocardiography. J Am Soc Echocardiogr. 2009;22(2):107-33.

[6]. Friesinger GC, Page EE, Ross RS: Prognostic significance of coronary arteriography. Trans Assoc Am Phys 83: 78, 1970.

[7]. Report of the Ad Hoc Committee for Grading of Coronary Artery Disease: A reporting system on patients evaluated for coronary artery disease. Council on Cardiovascular Surgery. Dallas, American Heart Association, document 73-315-A 